\title{
Elliptic problems on the ball endowed with Funk-type metrics
}

\author{
Alexandru Kristály ${ }^{*, * *} \&$ Imre J. Rudas** \\ *Department of Economics, Babeş-Bolyai University, Cluj-Napoca, Romania \\ Email address: alexandrukristaly@yahoo.com \\ **Institute of Applied Mathematics, Óbuda University, 1034 Budapest, Hungary
}

\begin{abstract}
We study Sobolev spaces on the $n$-dimensional unit ball $B^{n}(1)$ endowed with a parameter-depending Finsler metric $F_{a}, a \in[0,1]$, which interpolates between the Klein metric $(a=0)$ and Funk metric $(a=1)$, respectively. We show that the standard Sobolev space defined on the Finsler manifold $\left(B^{n}(1), F_{a}\right)$ is a vector space if and only if $a \in[0,1)$. Furthermore, by exploiting variational arguments, we provide non-existence and existence results for sublinear elliptic problems on $\left(B^{n}(1), F_{a}\right)$ involving the Finsler-Laplace operator whenever $a \in[0,1)$.
\end{abstract}

Keywords: Funk metric; Klein metric; Finsler-Laplace operator; Sobolev space; variational methods.

\section{INTRODUCTION}

The theory of Sobolev spaces on complete Riemannian manifolds is well understood and widely applied into the study of various elliptic problems, see e.g. Hebey [7]. Although Finsler geometry is a natural extension of Riemannian geometry, very little is known about Sobolev spaces on non-compact Finsler manifolds. One of the most important features of Finsler structures is that they can describe non-reversible phenomena. Such examples appear for instance in the Matsumoto mountain slope metric (describing the law of walking on a mountain under the action of gravity), the PoincaréFinsler disc model, the Funk metric on the $n$-dimensional unit ball $B^{n}(1) \subset \mathbb{R}^{n}$, etc.

The purpose of this paper is to study properties of the Sobolev space and some elliptic problems involving the Finsler-Laplace operator on $B^{n}(1) \subset \mathbb{R}^{n}$ which is endowed with a Funk-type metric. To be more precise, let $B^{n}(1)=\left\{x \in \mathbb{R}^{n}:|x|<1\right\}$ be the $n$-dimensional unit ball, $n \geq 2$, and for every $a \in[0,1]$, we consider the function $F_{a}: B^{n}(1) \times \mathbb{R}^{n} \rightarrow \mathbb{R}$ defined by

$$
F_{a}(x, y)=\frac{\sqrt{|y|^{2}-\left(|x|^{2}|y|^{2}-\langle x, y\rangle^{2}\right)}}{1-|x|^{2}}+a \frac{\langle x, y\rangle}{1-|x|^{2}}, x \in B^{n}(1), y \in T_{x} B^{n}(1)=\mathbb{R}^{n} .
$$

Hereafter, $|\cdot|$ and $\langle\cdot, \cdot\rangle$ denote the $n$-dimensional Euclidean norm and inner product. Standard arguments from Finsler geometry show that the pair $\left(B^{n}(1), F_{a}\right)$ is a Finsler manifold of Randers type. In fact, for $a=0$, the manifold $\left(B^{n}(1), F_{0}\right)$ reduces to the well known Riemannian Klein model; for $a=1,\left(B^{n}(1), F_{1}\right)$ is the usual Finslerian 
Funk model, see Cheng and Shen [3] and Shen [13]. We introduce the Sobolev space associated with $\left(B^{n}(1), F_{a}\right)$; namely, let $W_{0}^{1,2, a}\left(B^{n}(1)\right)$ be the closure of $C_{0}^{\infty}\left(B^{n}(1)\right)$ with respect to the (positively homogeneous) norm

$$
\|u\|_{W^{1,2, a}}=\left(\int_{B^{n}(1)} F_{a}^{* 2}(x, D u(x)) \mathrm{d} V_{F_{a}}(x)+\int_{B^{n}(1)} u^{2}(x) \mathrm{d} V_{F_{a}}(x)\right)^{1 / 2},
$$

where $F_{a}^{*}$ denotes the polar transform of $F_{a}$, and $\mathrm{d} V_{F_{a}}$ is the canonical Hausdorff measure on $\left(B^{n}(1), F_{a}\right)$; see Section 2 for details. For $a=0$ (Klein metric case), the space $W_{0}^{1,2,0}\left(B^{n}(1)\right)$ endowed with the (absolutely homogenous) norm $\|\cdot\|_{H_{1}^{2}}=$ $\|\cdot\|_{W^{1,2,0}}$ is nothing but the usual Sobolev space $H_{1}^{2}\left(B^{n}(1)\right)$ on the Riemannian manifold $\left(B^{n}(1), F_{0}\right)$ having a Hilbert structure, see Hebey [7]. Sobolev spaces on generic Finsler manifolds were introduced (and studied in the compact case) in [6] and [12].

Our first result reads as follows.

Theorem 1.1. Let $a \in[0,1]$. Then the following assertions are equivalent:

(i) $W_{0}^{1,2, a}\left(B^{n}(1)\right)$ is a vector space over $\mathbb{R}$;

(ii) $a \in[0,1)$.

The proof of Theorem 1.1 is based on the following two facts:

- when $a=1$, i.e., $F_{a}$ is the usual Funk metric, we construct a function $u \in$ $W_{0}^{1,2,1}\left(B^{n}(1)\right)$ such that $-u \notin W_{0}^{1,2,1}\left(B^{n}(1)\right)$

- when $a \in[0,1)$, the vector space structure of $W_{0}^{1,2, a}\left(B^{n}(1)\right)$ follows in a standard way by exploiting the convexity of $F_{a}^{*}$ and the finiteness of the reversibility constant of $\left(B^{n}(1), F_{a}\right)$. As a byproduct, the equivalence of the norms $\|\cdot\|_{W^{1,2, a}}$ and $\|\cdot\|_{H_{1}^{2}}$ easily follows.

In the second part of the paper we consider the highly nonlinear problem

$$
\begin{cases}-\Delta_{F_{a}} u=\lambda \kappa(x) g(u) & \text { in } \quad B^{n}(1) \\ u \rightarrow 0 & \text { if } \quad|x| \rightarrow 1\end{cases}
$$

where $a \in[0,1), \boldsymbol{\Delta}_{F_{a}}$ denotes the Finsler-Laplace operator associated with the Funktype metric $F_{a}$ on $B^{n}(1), \lambda \geq 0$ is a parameter, $\kappa \in L^{1}\left(B^{n}(1)\right) \cap L^{\infty}\left(B^{n}(1)\right)$ and $g:[0, \infty) \rightarrow \mathbb{R}$ is a continuous function. Note that when $a=0, \boldsymbol{\Delta}_{F_{a}}$ becomes the usual Laplace-Betrami operator $\Delta_{F_{0}}$ on the Klein ball model $\left(B^{n}(1), F_{0}\right)$; however, when $a \neq 0$, the operator $\boldsymbol{\Delta}_{F_{a}}$ is highly non-linear (neither additive nor absolutely homogeneous). On the continuous function $g$, we require

(g1) $g(s)=o(s)$ as $s \rightarrow 0^{+}$and $s \rightarrow \infty$;

(g2) $G\left(s_{0}\right)>0$ for some $s_{0}>0$, where $G(s)=\int_{0}^{s} g(t) d t$.

The assumption $g(s)=o(s)$ as $s \rightarrow \infty$ means that $g$ is sublinear at infinity. Moreover, due to (g1) and (g2), the number

$$
c_{g}=\max _{s>0} \frac{g(s)}{s}
$$

is well-defined and positive.

Our second result reads as follows. 
Theorem 1.2. Let $a \in[0,1), \kappa \in L^{1}\left(B^{n}(1)\right) \cap L^{\infty}\left(B^{n}(1)\right) \backslash\{0\}$ be a radially symmetric non-negative function, and a continuous function $g:[0, \infty) \rightarrow \mathbb{R}$ verifying (g1) and (g2). Then

(i) $\left(\mathcal{P}_{\lambda}\right)$ has only the zero solution whenever $0 \leq \lambda<c_{g}^{-1}\|\kappa\|_{L^{\infty}}^{-1} \frac{(n-1)^{2}\left(1-a^{2}\right)^{\frac{n+1}{2}}}{4(1+a)^{2}}$;

(ii) there exists $\tilde{\lambda}>0$ such that $\left(\mathcal{P}_{\lambda}\right)$ has at least two distinct non-zero, nonnegative, radially symmetric weak solutions whenever $\lambda>\tilde{\lambda}$.

The proof of (i) combines a direct computation with a result of Federer and Fleming [5] applied for the Klein ball model. In order to prove (ii), we shall exploit Theorem 1.1 together with variational arguments (minimization, mountain pass and the principle of symmetric criticality). In fact, Theorem 1.2 seems to be the first existence result within the class of elliptic problems on non-compact Finsler manifolds.

\section{Preliminaries}

2.1. Randers spaces. Let $M$ be a smooth, $n$-dimensional manifold and $T M=$ $\bigcup_{x \in M} T_{x} M$ be its tangent bundle. Throughout of this subsection, the function $F$ : $T M \rightarrow[0, \infty)$ is given by

$$
F(x, y)=\sqrt{h_{x}(y, y)}+\beta_{x}(y),(x, y) \in T M,
$$

where $h$ is a Riemannian metric on $M, \beta$ is an 1-form on $M$, and we assume that

$$
\|\beta\|_{h}(x)=\sqrt{h_{x}^{*}\left(\beta_{x}, \beta_{x}\right)}<1, \forall x \in M .
$$

Here, the co-metric $h_{x}^{*}$ can be identified by the inverse of the symmetric, positive definite matrix $h_{x}$. The pair $(M, F)$ is a Randers space which is a typical Finsler manifold, i.e., the following properties hold:

(a) $F \in C^{\infty}(T M \backslash\{0\})$;

(b) $F(x, t y)=t F(x, y)$ for all $t \geq 0$ and $(x, y) \in T M$;

(c) $g_{(x, y)}=\left[g_{i j}(x, y)\right]:=\left[\frac{1}{2} F^{2}(x, y)\right]_{y^{i} y^{j}}$ is positive definite for all $(x, y) \in T M \backslash\{0\}$, see Bao, Chern and Shen [1]. Clearly, the Randers metric $F$ in (2.1) is symmetric, i.e., $F(x,-y)=F(x, y)$ for every $(x, y) \in T M$, if and only if $\beta=0$.

Unlike the Levi-Civita connection in the Riemannian case, there is no unique natural connection in the Finsler geometry. Among these connections on the pull-back bundle $\pi^{*} T M$, we choose a torsion free and almost metric-compatible linear connection on $\pi^{*} T M$, the so-called Chern connection, see Bao, Chern and Shen [1, Theorem 2.4.1]. Since the notions of geodesics, forward/backward completeness and flag curvature will not be explicitly used in the sequel, we assume the reader is familiar with them; for details, see [1].

Let $\sigma:[0, r] \rightarrow M$ be a piecewise $C^{\infty}$ curve. The value $L_{F}(\sigma)=\int_{0}^{r} F(\sigma(t), \dot{\sigma}(t)) \mathrm{d} t$ denotes the integral length of $\sigma$. For $x_{1}, x_{2} \in M$, denote by $\Lambda\left(x_{1}, x_{2}\right)$ the set of all piecewise $C^{\infty}$ curves $\sigma:[0, r] \rightarrow M$ such that $\sigma(0)=x_{1}$ and $\sigma(r)=x_{2}$. Define the 
distance function $d_{F}: M \times M \rightarrow[0, \infty)$ by

$$
d_{F}\left(x_{1}, x_{2}\right)=\inf _{\sigma \in \Lambda\left(x_{1}, x_{2}\right)} L_{F}(\sigma)
$$

One clearly has that $d_{F}\left(x_{1}, x_{2}\right)=0$ if and only if $x_{1}=x_{2}$, and $d_{F}$ verifies the triangle inequality.

The Hausdorff volume form $\mathrm{d} V_{F}$ on the Randers space $(M, F)$ is given by

$$
\mathrm{d} V_{F}(x)=\left(1-\|\beta\|_{h}^{2}(x)\right)^{\frac{n+1}{2}} \mathrm{~d} V_{h}(x),
$$

where $\mathrm{d} V_{h}(x)$ denotes the usual Riemannian volume form of $h$ on $M$, see Cheng and Shen 3 .

For every $(x, \alpha) \in T^{*} M$, the polar transform (or, co-metric) of $F$ from (2.1) is

$$
\begin{aligned}
F^{*}(x, \alpha) & \stackrel{\text { def. }}{=} \sup _{y \in T_{x} M \backslash\{0\}} \frac{\alpha(y)}{F(x, y)} \\
& =\frac{\sqrt{h_{x}^{* 2}(\alpha, \beta)+\left(1-\|\beta\|_{h}^{2}(x)\right)\|\alpha\|_{h}^{2}(x)}-h_{x}^{*}(\alpha, \beta)}{1-\|\beta\|_{h}^{2}(x)} .
\end{aligned}
$$

Let $u: M \rightarrow \mathbb{R}$ be a differentiable function in the distributional sense. The gradient of $u$ is defined by

$$
\nabla_{F} u(x)=J^{*}(x, D u(x)),
$$

where $J^{*}: T^{*} M \rightarrow T M$ is the Legendre transform

$$
J^{*}(x, \alpha)=\sum_{i=1}^{n} \frac{\partial}{\partial \alpha_{i}}\left(\frac{1}{2} F^{* 2}(x, \alpha)\right) \frac{\partial}{\partial x^{i}},
$$

and $D u(x) \in T_{x}^{*} M$ denotes the (distributional) derivative of $u$ at $x \in M$. In local coordinates, one has

$$
\begin{gathered}
D u(x)=\sum_{i=1}^{n} \frac{\partial u}{\partial x^{i}}(x) \mathrm{d} x^{i}, \\
\nabla_{F} u(x)=\sum_{i, j=1}^{n} g_{i j}^{*}(x, D u(x)) \frac{\partial u}{\partial x^{i}}(x) \frac{\partial}{\partial x^{j}} .
\end{gathered}
$$

In general, $u \mapsto \nabla_{F} u$ is not linear. The mean value theorem implies that

$$
(D u(x)-D v(x))\left(\nabla_{F} u(x)-\nabla_{F} v(x)\right) \geq l_{F} F^{* 2}(D u(x)-D v(x)), \forall x \in M,
$$

where

$$
\begin{aligned}
l_{F} & \stackrel{\text { def. }}{=} \inf _{x \in M} \inf _{y, v, w \in T_{x} M \backslash\{0\}} \frac{g_{(x, v)}(y, y)}{g_{(x, w)}(y, y)} \\
& =\inf _{x \in M}\left(\frac{1-\|\beta\|_{h}(x)}{1+\|\beta\|_{h}(x)}\right)^{2} .
\end{aligned}
$$


If $x_{0} \in M$ is fixed, then due to [12], one has $(2.9)$

$F^{*}\left(x, D d_{F}\left(x_{0}, x\right)\right)=F\left(x, \nabla_{F} d_{F}\left(x_{0}, x\right)\right)=D d_{F}\left(x_{0}, x\right)\left(\nabla_{F} d_{F}\left(x_{0}, x\right)\right)=1$ for a.e. $x \in M$.

Let $X$ be a vector field on $M$. In a local coordinate system $\left(x^{i}\right)$, the divergence is defined by $\operatorname{div}_{F}(X)=\frac{1}{\sigma_{F}} \frac{\partial}{\partial x^{i}}\left(\sigma_{F} X^{i}\right)$, where $\sigma_{F}(x)=\frac{\omega_{n}}{\operatorname{Vol}\left(B_{x}(1)\right)}, \omega_{n}$ and $\operatorname{Vol}\left(B_{x}(1)\right)$ being the Euclidean volumes of the unit ball $B^{n}(1)$ and of the unit tangent ball $B_{x}(1)=$ $\left\{y=\left(y^{i}\right): F\left(x, y^{i} \partial / \partial x^{i}\right)<1\right\}$, respectively. The Finsler-Laplace operator

$$
\boldsymbol{\Delta}_{F} u=\operatorname{div}_{F}\left(\nabla_{F} u\right)
$$

acts on $W_{\text {loc }}^{1,2}(M)$ and for every $v \in C_{0}^{\infty}(M)$,

$$
\int_{M} v \boldsymbol{\Delta}_{F} u \mathrm{~d} V_{F}(x)=-\int_{M} D v\left(\nabla_{F} u\right) \mathrm{d} V_{F}(x),
$$

see [12] and [14]. In particular, if $\beta=0$ (thus, the Randers space $(M, F)$ reduces to the Riemannian manifold $(M, h))$ the Finsler-Laplace operator becomes the usual Laplace-Beltrami operator $\Delta_{h} u$.

By definition, the reversibility constant associated with $F$ is given by the formula

$$
r_{F} \stackrel{\text { def. }}{=} \sup _{x \in M} r_{F}(x)
$$

where

$$
\begin{aligned}
r_{F}(x) \stackrel{\text { def. }}{=} \sup _{y \in T_{x} M \backslash\{0\}} \frac{F(x, y)}{F(x,-y)} \\
=\frac{1+\|\beta\|_{h}(x)}{1-\|\beta\|_{h}(x)},
\end{aligned}
$$

see Rademacher [10]. It is clear that $r_{F} \geq 1$ (possibly, $r_{F}=+\infty$ ) and $r_{F}=1$ if and only if $(M, F)$ is reversible, i.e., $\beta=0$.

2.2. The Funk-type metric $F_{a}$ on $B^{n}(1)$. In this subsection, we explicitly compute the objects introduced in the previous subsection for the Funk-type metric $F_{a}$ on $B^{n}(1)$, $a \in[0,1]$, given in (1.1). Therefore, one has $M=B^{n}(1)$ and the Randers metric $F_{a}$ is coming from the Klein metric $h_{K}$,

$$
\left(h_{K}\right)_{x}(y, y)=\frac{\sqrt{|y|^{2}-\left(|x|^{2}|y|^{2}-\langle x, y\rangle^{2}\right)}}{1-|x|^{2}}
$$

and by the 1 -form

$$
\beta_{x}=a \frac{x}{1-|x|^{2}}
$$

It is clear that

$$
\left(h_{K}\right)_{i j}=\frac{\delta_{i j}}{1-|x|^{2}}+\frac{x_{i} x_{j}}{\left(1-|x|^{2}\right)^{2}}, i, j \in\{1, \ldots, n\},
$$

and according to Cheng and Shen [3, Lemma 1.1.1], we have that $h_{K}^{*}=\left(h_{K}\right)^{-1}$ where the elements of the matrix are given by

$$
h_{K}^{i j}=\left(1-|x|^{2}\right)\left(\delta_{i j}-x_{i} x_{j}\right), i, j \in\{1, \ldots, n\} .
$$


Consequently,

$$
\|\beta\|_{h_{K}}(x)=\sqrt{h_{K}^{i j}\left(\beta_{x}^{i}, \beta_{x}^{j}\right)}=a|x| .
$$

Therefore, the reversibility constant associated with $F_{a}$ on $B^{n}(1)$ is given by

$$
r_{F_{a}}=\left\{\begin{array}{lll}
\frac{1+a}{1-a} & \text { if } & a \in[0,1) \\
+\infty & \text { if } & a=1
\end{array}\right.
$$

Due to (2.3), we have

$$
\mathrm{d} V_{F_{a}}(x)=\left(1-a^{2}|x|^{2}\right)^{\frac{n+1}{2}} \mathrm{~d} V_{h_{K}}(x),
$$

where the Klein volume form is given by

$$
\mathrm{d} V_{h_{K}}(x)=\frac{1}{\left(1-|x|^{2}\right)^{\frac{n+1}{2}}} \mathrm{~d} x .
$$

Finally, the polar transform of $F_{a}$ is

$$
F_{a}^{*}(x, y)=\frac{\sqrt{\left(1-|x|^{2}\right)\left(1-a^{2}|x|^{2}\right)|y|^{2}-\left(1-a^{2}\right)\left(1-|x|^{2}\right)\langle x, y\rangle^{2}}-a\left(1-|x|^{2}\right)\langle x, y\rangle}{1-a^{2}|x|^{2}} .
$$

It is clear that $F_{a}^{* *}=F_{a}$ and $r_{F_{a}^{*}}=r_{F_{a}}$.

\section{The Sobolev space on $\left(B^{n}(1), F_{a}\right)$ : Proof of Theorem 1.1}

Proof of Theorem 1.1. "(ii) $\Rightarrow(\mathrm{i})$." Let $a \in[0,1)$. Due to the convexity of $F_{a}^{* 2}$, if $u, v \in W_{0}^{1,2, a}\left(B^{n}(1)\right)$ then $u+v \in W_{0}^{1,2, a}\left(B^{n}(1)\right)$. Since $r_{F_{a}^{*}}=\frac{1+a}{1-a}$ is finite, one also has that $c u \in W_{0}^{1,2, a}\left(B^{n}(1)\right)$ for every $c \in \mathbb{R}$ and $u \in W_{0}^{1,2, a}\left(B^{n}(1)\right)$. The other properties of a vector space are trivially verified.

"(i) $\Rightarrow$ (ii)." The space $W_{0}^{1,2, a}\left(B^{n}(1)\right)$ is assumed to be a vector space over $\mathbb{R}$; by contradiction, we also assume that one may have $a=1$. In this case, $F_{a}$ is precisely the Funk metric

$$
F_{1}(x, y)=\frac{\sqrt{|y|^{2}-\left(|x|^{2}|y|^{2}-\langle x, y\rangle^{2}\right)}}{1-|x|^{2}}+\frac{\langle x, y\rangle}{1-|x|^{2}}, x \in B^{n}(1), y \in \mathbb{R}^{n} .
$$

Note that the metric $F_{1}$ can be obtained by

$$
\left|x+\frac{y}{F_{1}(x, y)}\right|=1
$$

while the distance function associated to $F_{1}$ is given by

$$
d_{F_{1}}\left(x_{1}, x_{2}\right)=\ln \frac{\sqrt{\left|x_{1}-x_{2}\right|^{2}-\left(\left|x_{1}\right|^{2}\left|x_{2}\right|^{2}-\left\langle x_{1}, x_{2}\right\rangle^{2}\right)}-\left\langle x_{1}, x_{2}-x_{1}\right\rangle}{\sqrt{\left|x_{1}-x_{2}\right|^{2}-\left(\left|x_{1}\right|^{2}\left|x_{2}\right|^{2}-\left\langle x_{1}, x_{2}\right\rangle^{2}\right)}-\left\langle x_{2}, x_{2}-x_{1}\right\rangle}, x_{1}, x_{2} \in B^{n}(1),
$$

see Shen [13, p.141 and p.4]. In particular,

$$
d_{F_{1}}(0, x)=-\ln (1-|x|), x \in B^{n}(1) .
$$


First, by (2.9) or direct checking via (2.15), we have that

$$
F_{1}^{*}\left(x, D d_{F_{1}}(0, x)\right)=1 .
$$

Second, a direct computation and (2.15) shows that

$$
F_{1}^{*}\left(x,-D d_{F_{1}}(0, x)\right)=\frac{1+|x|}{1-|x|} .
$$

Let $u: B^{n}(1) \rightarrow \mathbb{R}$ be defined by $u(x)=-\sqrt{1-|x|}=-e^{-\frac{d_{F_{1}}(0, x)}{2}}$. It is clear that $u \in W_{\text {loc }}^{1,2}\left(B^{n}(1)\right)$. First, since $\mathrm{d} V_{F_{1}}(x)=\mathrm{d} x$, we have

$$
\int_{B^{n}(1)} u^{2}(x) \mathrm{d} V_{F_{1}}(x)=\frac{\omega_{n}}{n+1} .
$$

On one hand, since $D u(x)=\frac{1}{2} e^{-\frac{d_{F_{1}}(0, x)}{2}} D d_{F_{1}}(0, x)$, by (3.1) it yields

$$
\begin{aligned}
C_{1} & :=\int_{B^{n}(1)} F_{1}^{* 2}(x, D u(x)) \mathrm{d} V_{F_{1}}(x)=\frac{1}{4} \int_{B^{n}(1)}(1-|x|) \mathrm{d} x \\
& =\frac{\omega_{n}}{4(n+1)} .
\end{aligned}
$$

Therefore, $\|u\|_{W^{1,2,1}}^{2}=\frac{5 \omega_{n}}{4(n+1)}$, so $u \in W_{0}^{1,2,1}\left(B^{n}(1)\right)$.

On the other hand, relation (3.2) implies that

$$
\begin{aligned}
C_{2} & :=\int_{B^{n}(1)} F_{1}^{* 2}(x,-D u(x)) \mathrm{d} V_{F_{1}}(x)=\frac{1}{4} \int_{B^{n}(1)} \frac{(1+|x|)^{2}}{1-|x|} \mathrm{d} x \\
& =+\infty
\end{aligned}
$$

i.e., $-u \notin W_{0}^{1,2,1}\left(B^{n}(1)\right)$, contradicting our initial assumption.

Remark 3.1. Let $a \in[0,1)$. For every $x \in B^{n}(1)$, one has $0<1-a^{2} \leq 1-a^{2}|x|^{2} \leq 1$; thus, the volume forms $\mathrm{d} V_{F_{a}}(x)$ and $\mathrm{d} V_{h_{K}}(x)$ generate equivalent measures. Moreover, one also has

$$
\frac{1}{(1+a)^{2}} h_{K}^{*}(y, y) \leq F_{a}^{* 2}(x, y) \leq \frac{1}{(1-a)^{2}} h_{K}^{*}(y, y), x \in B^{n}(1), y \in \mathbb{R}^{n} .
$$

Consequently,

$$
\frac{\left(1-a^{2}\right)^{\frac{n+1}{4}}}{1+a}\|u\|_{H_{1}^{2}} \leq\|u\|_{W^{1,2, a}} \leq \frac{1}{1-a}\|u\|_{H_{1}^{2}}, u \in C_{0}^{\infty}\left(B^{n}(1)\right) .
$$

In particular, the topologies generated by the objects $\left(W_{0}^{1,2, a}\left(B^{n}(1)\right),\|\cdot\|_{W^{1,2, a}}\right)$ and $\left(H_{1}^{2}\left(B^{n}(1)\right),\|\cdot\|_{H_{1}^{2}}\right)$ are equivalent whenever $a \in[0,1)$. Moreover, a result of Federer and Fleming [5] for the Klein ball model $\left(B^{n}(1), F_{0}\right)$ states that

$$
\int_{B^{n}(1)} u^{2}(x) \mathrm{d} V_{h_{K}}(x) \leq \frac{4}{(n-1)^{2}} \int_{B^{n}(1)} h_{K}^{*}(D u(x), D u(x)) \mathrm{d} V_{h_{K}}(x), \forall C_{0}^{\infty}\left(B^{n}(1)\right) .
$$


Therefore, the norm $\|\cdot\|_{H_{1}^{2}}$ and the 'gradient' norm over the Klein metric model given by

$$
u \mapsto\|u\|_{K}=\left(\int_{B^{n}(1)} h_{K}^{*}(D u(x), D u(x)) \mathrm{d} V_{h_{K}}(x)\right)^{\frac{1}{2}}
$$

are also equivalent, i.e.,

$$
\|u\|_{K} \leq\|u\|_{H_{1}^{2}} \leq\left(1+\frac{4}{(n-1)^{2}}\right)^{\frac{1}{2}}\|u\|_{K}
$$

Remark 3.2. The space $W_{0}^{1,2,1}\left(B^{n}(1)\right)$ is a closed and convex cone in $L_{1}^{2}\left(B^{n}(1)\right)$, where $L_{a}^{p}\left(B^{n}(1)\right)$ denotes the usual class of measurable functions $u: B^{n}(1) \rightarrow \mathbb{R}$ such that

$$
\|u\|_{L_{a}^{p}}=\left(\int_{B^{n}(1)}|u(x)|^{p} \mathrm{~d} V_{F_{a}}(x)\right)^{\frac{1}{p}}<\infty
$$

whenever $1 \leq p<\infty$. $L_{0}^{p}$ will be denoted in the usual way by $L^{p}$. $L^{\infty}\left(B^{n}(1)\right)$ denotes the class of essentially bounded functions on $B^{n}(1)$ with the usual sup-norm $\|\cdot\|_{L^{\infty}}$.

\section{Problem $\left(\mathcal{P}_{\lambda}\right)$ : Proof of Theorem 1.2}

Due to (g1), it follows that $g(0)=0$. Therefore, one can extend the function $g$ to $\mathbb{R}$ by $g(s)=0$ for $s \leq 0$; this extension will be considered throughout of this section.

An element $u \in W_{0}^{1,2, a}\left(B^{n}(1)\right)$ is a weak solution of problem $\left(\mathcal{P}_{\lambda}\right)$ if $u(x) \rightarrow 0$ as $|x| \rightarrow 1$ and

$$
\int_{B^{n}(1)} D v\left(\nabla_{F_{a}} u\right) \mathrm{d} V_{F_{a}}(x)=\lambda \int_{B^{n}(1)} \kappa(x) g(u(x)) v(x) \mathrm{d} V_{F_{a}}(x), \forall v \in C_{0}^{\infty}\left(B^{n}(1)\right) .
$$

Proof of Theorem 1.2. (i) Let $u \in W_{0}^{1,2, a}\left(B^{n}(1)\right)$ be a weak solution of $\left(\mathcal{P}_{\lambda}\right)$. By density reasons, in (4.1) we may use $v=u$ as a test-function, obtaining by (2.14), (3.4) and $(3.3)$ that

$$
\begin{aligned}
\int_{B^{n}(1)} F_{a}^{* 2}(x, D u(x)) \mathrm{d} V_{F_{a}}(x) & =\int_{B^{n}(1)} D u\left(\nabla_{F_{a}} u\right) \mathrm{d} V_{F_{a}}(x) \\
& =\lambda \int_{B^{n}(1)} \kappa(x) g(u(x)) u(x) \mathrm{d} V_{F_{a}}(x) \\
& \leq \lambda c_{g}\|\kappa\|_{L^{\infty}} \int_{B^{n}(1)} u^{2}(x) \mathrm{d} V_{h_{K}}(x) \\
& \leq \frac{4 \lambda c_{g}\|\kappa\|_{L^{\infty}}}{(n-1)^{2}} \int_{B^{n}(1)} h_{K}^{*}(D u(x), D u(x)) \mathrm{d} V_{h_{K}}(x) \\
& \leq \frac{4 \lambda c_{g}\|\kappa\|_{L^{\infty}}(1+a)^{2}}{(n-1)^{2}\left(1-a^{2}\right)^{\frac{n+1}{2}}} \int_{B^{n}(1)} F_{a}^{* 2}(x, D u(x)) \mathrm{d} V_{F_{a}}(x) .
\end{aligned}
$$

Consequently, if $0 \leq \lambda<c_{g}^{-1}\|\kappa\|_{L^{\infty}}^{-1} \frac{(n-1)^{2}\left(1-a^{2}\right)^{\frac{n+1}{2}}}{4(1+a)^{2}}, u$ is necessarily 0 .

(ii) The proof is divided into several steps. 
STEP 1. (Variational setting) Due to Remark 3.1, we may consider the energy functional $\mathcal{J}_{\lambda}: H_{1}^{2}\left(B^{n}(1)\right) \rightarrow \mathbb{R}$ associated with $\left(\mathcal{P}_{\lambda}\right)$, i.e.,

$$
\mathcal{J}_{\lambda}(u)=\frac{1}{2} \mathcal{E}(u)-\lambda \mathcal{G}(u)
$$

where

$$
\mathcal{E}(u)=\int_{B^{n}(1)} F_{a}^{* 2}(x, D u(x)) \mathrm{d} V_{F_{a}}(x) \text { and } \mathcal{G}(u)=\int_{B^{n}(1)} \kappa(x) G(u(x)) \mathrm{d} V_{F_{a}}(x) .
$$

On account of $(\mathrm{g} 1)$, the functional $\mathcal{J}_{\lambda}$ is well-defined and of class $C^{1}$; moreover, by (2.6), we have that

$$
\mathcal{J}_{\lambda}^{\prime}(u)(v)=\int_{B^{n}(1)}\left[D v\left(\nabla_{F_{a}} u\right)(x)-\lambda \kappa(x) g(u(x)) v(x)\right] \mathrm{d} V_{F_{a}}(x) .
$$

In particular, $\mathcal{J}_{\lambda}^{\prime}(u)=0$ if and only if (4.1) holds.

STEP 2. (Symmetrization) Although $H_{1}^{2}\left(B^{n}(1)\right)$ can be embedded into the Lebesgue space $L^{p}\left(B^{n}(1)\right), p \in\left[2,2^{*}\right)$, see Hebey [7, Proposition 3.7], this embedding is not compact. Thus, we consider the space of radially symmetric functions in $H_{1}^{2}\left(B^{n}(1)\right)$, i.e.,

$$
H_{r}\left(B^{n}(1)\right)=\left\{u \in H_{1}^{2}\left(B^{n}(1)\right): u(x)=u(|x|)\right\} .
$$

By using a Strauss-type inequality, Bhakta and Sandeep [2] proved that the embedding $H_{r}\left(B^{n}(1)\right) \hookrightarrow L^{p}\left(B^{n}(1)\right)$ is compact for every $p \in\left(2,2^{*}\right)$. [Note that in [2] the Poincaré ball model is used which is conformally equivalent to the Klein ball model.] Moreover, for every $u \in H_{r}\left(B^{n}(1)\right)$, the Strauss-estimate shows that $u(x) \rightarrow 0$ as $|x| \rightarrow 1$.

If we introduce the action of the orthogonal group $O(n)$ on $H_{1}^{2}\left(B^{n}(1)\right)$ in the usual manner, i.e.,

$$
(\tau u)(x)=u\left(\tau^{-1} x\right), u \in H_{1}^{2}\left(B^{n}(1)\right), \tau \in O(n), x \in B^{n}(1),
$$

then the fixed point set of $O(n)$ on $H_{1}^{2}\left(B^{n}(1)\right)$ is precisely the space $H_{r}\left(B^{n}(1)\right)$. Moreover, by using (2.15), let us observe that $F_{a}^{*}$ is $O(n)$-invariant, i.e.,

$$
F_{a}^{*}(\tau x, \tau y)=F_{a}^{*}(x, y), \forall \tau \in O(n), x \in B^{n}(1), y \in \mathbb{R}^{n} .
$$

Therefore, since $D(\tau u)(x)=\left(\tau^{-1}\right)^{t} D u\left(\tau^{-1} x\right)=\tau D u\left(\tau^{-1} x\right)$, where ${ }^{t}$ denotes the transpose of a matrix, we have for every $\tau \in O(n)$ and $u \in H_{1}^{2}\left(B^{n}(1)\right)$ that

$$
\begin{aligned}
\mathcal{E}(\tau u) & =\int_{B^{n}(1)} F_{a}^{* 2}(x, D(\tau u)(x)) \mathrm{d} V_{F_{a}}(x) \\
& \left.=\int_{B^{n}(1)} F_{a}^{* 2}\left(x, \tau D u\left(\tau^{-1} x\right)\right) \mathrm{d} V_{F_{a}}(x) \quad \text { (change of var. } \tau^{-1} x=z\right) \\
& \left.=\int_{B^{n}(1)} F_{a}^{* 2}(\tau z, \tau D u(z)) \mathrm{d} V_{F_{a}}(\tau z) \quad \text { (see (4.2) and } \mathrm{d} V_{F_{a}}(\tau z)=\mathrm{d} V_{F_{a}}(z)\right) \\
& =\int_{B^{n}(1)} F_{a}^{* 2}(z, D u(z)) \mathrm{d} V_{F_{a}}(z) \\
& =\mathcal{E}(u),
\end{aligned}
$$


i.e., $\mathcal{E}$ is $O(n)$-invariant. Similar reasoning as above shows that $\mathcal{G}$ is also $O(n)$-invariant and $O(n)$ act isometrically on $H_{1}^{2}\left(B^{n}(1)\right)$, i.e.,

$$
\mathcal{G}(\tau u)=\mathcal{G}(g u) \text { and }\|\tau u\|_{H_{1}^{2}}=\|u\|_{H_{1}^{2}}, \forall \tau \in O(n), u \in H_{1}^{2}\left(B^{n}(1)\right) .
$$

By the above properties it follows that $\mathcal{J}_{\lambda}$ is $O(n)$-invariant. Therefore, the principle of symmetric criticality of Palais (see Kristály, Rădulescu and Varga [9, Theorem 1.50]) implies that the critical points of

$$
\mathcal{R}_{\lambda}=\left.\mathcal{J}_{\lambda}\right|_{H_{r}\left(B^{n}(1)\right)}
$$

are also critical points for the original functional $\mathcal{J}_{\lambda}$. In addition, since $u(x) \rightarrow 0$ as $|x| \rightarrow 1$ for every $u \in H_{r}\left(B^{n}(1)\right)$, we conclude that it is enough to guarantee critical points for the functional $\mathcal{R}_{\lambda}$ in order to find radially symmetric, weak solutions for problem $\left(\mathcal{P}_{\lambda}\right)$.

For simplicity, let $\mathcal{E}_{r}$ and $\mathcal{G}_{r}$ be the restrictions of $\mathcal{E}$ and $\mathcal{G}$ to $H_{r}\left(B^{n}(1)\right)$, respectively. In the sequel, we shall show that there are at least two critical points for $\mathcal{R}_{\lambda}$ whenever $\lambda$ belongs to a suitable interval.

STEP 3. (Subquadraticity of $\mathcal{G}_{r}$ ) We claim that

$$
\lim _{\substack{u \in H_{r}\left(B^{n}(1)\right) \\\|u\|_{H_{1}^{2} \rightarrow 0}}} \frac{\mathcal{G}_{r}(u)}{\|u\|_{H_{1}^{2}}^{2}}=\lim _{\substack{u \in H_{r}\left(B^{n}(1)\right) \\\|u\|_{H_{1}^{2}} \rightarrow \infty}} \frac{\mathcal{G}_{r}(u)}{\|u\|_{H_{1}^{2}}^{2}}=0 .
$$

Due to (g1), for every $\varepsilon>0$ there exists $\delta_{\varepsilon} \in(0,1)$ such that

$$
0 \leq|g(s)| \leq \frac{\varepsilon}{\|\kappa\|_{L^{\infty}}}|s| \text { for all }|s| \leq \delta_{\varepsilon} \text { and }|s| \geq \delta_{\varepsilon}^{-1},
$$

Fix $p \in\left(2,2^{*}\right)$; clearly, the function $s \mapsto \frac{g(s)}{s^{p-1}}$ is bounded on $\left[\delta_{\varepsilon}, \delta_{\varepsilon}^{-1}\right]$. Therefore, for some $m_{\varepsilon}>0$, one has that

$$
0 \leq|g(s)| \leq \frac{\varepsilon}{\|\kappa\|_{L^{\infty}}}|s|+m_{\varepsilon}|s|^{p-1} \text { for all } s \in \mathbb{R} .
$$

Thus, for every $u \in H_{r}\left(B^{n}(1)\right)$, it yields that

$$
\begin{aligned}
0 \leq\left|\mathcal{G}_{r}(u)\right| & \leq \int_{B^{n}(1)} \kappa(x)|G(u(x))| \mathrm{d} V_{F_{a}}(x) \\
& \leq \int_{B^{n}(1)} \kappa(x)\left[\frac{\varepsilon}{2\|\kappa\|_{L^{\infty}}} u(x)^{2}+\frac{m_{\varepsilon}}{p}|u(x)|^{p}\right] \mathrm{d} V_{h_{K}}(x) \\
& \leq \int_{B^{n}(1)}\left[\frac{\varepsilon}{2} u(x)^{2}+\frac{m_{\varepsilon}}{p} \kappa(x)|u(x)|^{p}\right] \mathrm{d} V_{h_{K}}(x) \\
& \leq \frac{\varepsilon}{2}\|u\|_{H_{1}^{2}}^{2}+\frac{m_{\varepsilon}}{p}\|\kappa\|_{L^{\infty}} S_{p}^{p}\|u\|_{H_{1}^{2}}^{p},
\end{aligned}
$$

where $S_{p}>0$ is the best embedding constant in $H_{r}\left(B^{n}(1)\right) \hookrightarrow L^{p}\left(B^{n}(1)\right)$. Thus, for every $u \in H_{r}\left(B^{n}(1)\right) \backslash\{0\}$,

$$
0 \leq \frac{\left|\mathcal{G}_{r}(u)\right|}{\|u\|_{H_{1}^{2}}^{2}} \leq \frac{\varepsilon}{2}+\frac{m_{\varepsilon}}{p}\|\kappa\|_{L^{\infty}} S_{p}^{p}\|u\|_{H_{1}^{2}}^{p-2} .
$$


Since $p>2$ and $\varepsilon>0$ is arbitrarily small, the first limit in (4.3) follows once $\|u\|_{H_{1}^{2}} \rightarrow 0$ in $H_{r}\left(B^{n}(1)\right)$.

Let $q \in(1,2)$. Since $g \in C(\mathbb{R}, \mathbb{R})$, there also exists a number $M_{\varepsilon}>0$ such that

$$
0 \leq \frac{|g(s)|}{s^{q-1}} \leq M_{\varepsilon} \text { for all } s \in\left[\delta_{\varepsilon}, \delta_{\varepsilon}^{-1}\right]
$$

where $\delta_{\varepsilon} \in(0,1)$ is from (4.4). The latter relation together with (4.4) give that

$$
0 \leq|g(s)| \leq \frac{\varepsilon}{\|\kappa\|_{L^{\infty}}}|s|+M_{\varepsilon}|s|^{q-1} \text { for all } s \in \mathbb{R}
$$

Similarly as above, it yields that

$$
0 \leq\left|\mathcal{G}_{r}(u)\right| \leq \frac{\varepsilon}{2}\|u\|_{H_{1}^{2}}^{2}+\frac{M_{\varepsilon}}{q}\|\kappa\|_{L^{\frac{2}{2-q}}}\|u\|_{H_{1}^{2}}^{q} .
$$

For every $u \in H_{r}\left(B^{n}(1)\right) \backslash\{0\}$, we have that

$$
0 \leq \frac{\left|\mathcal{G}_{r}(u)\right|}{\|u\|_{H_{1}^{2}}^{2}} \leq \frac{\varepsilon}{2}+\frac{M_{\varepsilon}}{q}\|\kappa\|_{L^{\frac{2}{2-q}}}\|u\|_{H_{1}^{2}}^{q-2} .
$$

Since $\varepsilon>0$ is arbitrary and $q \in(1,2)$, taking the limit $\|u\|_{H_{1}^{2}} \rightarrow \infty$ in $H_{r}\left(B^{n}(1)\right)$, we obtain the second relation in (4.3).

STEP 4. (Properties of $\mathcal{R}_{\lambda}$ ) We are going to prove that the functional $\mathcal{R}_{\lambda}$ is bounded from below, coercive, and verifies the Palais-Smale condition on $H_{r}\left(B^{n}(1)\right)$ for every $\lambda \geq 0$. First, by (4.6), it follows that

$$
\begin{aligned}
\mathcal{R}_{\lambda}(u) & =\frac{1}{2} \mathcal{E}_{r}(u)-\lambda \mathcal{G}_{r}(u) \\
& \geq \frac{\left(1-a^{2}\right)^{\frac{n+1}{2}}}{2(1+a)^{2}}\|u\|_{K}^{2}-\lambda \frac{\varepsilon}{2}\|u\|_{H_{1}^{2}}^{2}-\lambda \frac{M_{\varepsilon}}{r}\|\kappa\|_{L^{\frac{2}{2-r}}}\|u\|_{H_{1}^{2}}^{r}
\end{aligned}
$$

Choosing $\varepsilon>0$ sufficiently small, since $\|\cdot\|_{H_{1}^{2}}$ and $\|\cdot\|_{K}$ are equivalent norms (see (3.5) ) and $r<2$, it follows that $\mathcal{R}_{\lambda}$ is bounded from below and coercive.

Now, let $\left\{u_{k}\right\}$ be a sequence in $H_{r}\left(B^{n}(1)\right)$ such that $\left\{\mathcal{R}_{\lambda}\left(u_{k}\right)\right\}$ is bounded and $\left\|\mathcal{R}_{\lambda}^{\prime}\left(u_{k}\right)\right\|_{*} \rightarrow 0$. Since $\mathcal{R}_{\lambda}$ is coercive, the sequence $\left\{u_{k}\right\}$ is bounded in $H_{r}\left(B^{n}(1)\right)$. Therefore, up to a subsequence, we may suppose that $u_{k} \rightarrow u$ weakly in $H_{r}\left(B^{n}(1)\right)$ and $u_{k} \rightarrow u$ strongly in $L^{p}\left(B^{n}(1)\right)$ for some $u \in H_{r}\left(B^{n}(1)\right)$ and $p \in\left(2,2^{*}\right)$. In particular, we have that

$$
\mathcal{R}_{\lambda}^{\prime}(u)\left(u-u_{k}\right) \rightarrow 0 \text { and } R_{\lambda}^{\prime}\left(u_{k}\right)\left(u-u_{k}\right) \rightarrow 0 \text { as } k \rightarrow \infty .
$$

A direct computation gives that

$$
\begin{gathered}
\int_{B^{n}(1)}\left(D u(x)-D u_{k}(x)\right)\left(\nabla_{F} u(x)-\nabla_{F} u_{k}(x)\right) \mathrm{d} V_{F_{a}}(x)= \\
=\mathcal{R}_{\lambda}^{\prime}(u)\left(u-u_{k}\right)-R_{\lambda}^{\prime}\left(u_{k}\right)\left(u-u_{k}\right)+\lambda \int_{B^{n}(1)} \kappa(x)\left[g\left(u_{k}\right)-g(u)\right]\left(u_{k}-u\right) \mathrm{d} V_{F_{a}}(x) .
\end{gathered}
$$


By (4.7), the first two terms tend to zero. Moreover, due to (4.5), it follows that

$$
\begin{aligned}
T:= & \int_{B^{n}(1)} \kappa(x)\left|g\left(u_{k}\right)-g(u)\right| \cdot\left|u_{k}-u\right| \mathrm{d} V_{F_{a}}(x) \\
\leq & \int_{B^{n}(1)}\left(\varepsilon\left(\left|u_{k}\right|+|u|\right)+m_{\varepsilon}\|\kappa\|_{L^{\infty}}\left(\left|u_{n}\right|^{p-1}+|u|^{p-1}\right)\right)\left|u_{k}-u\right| \mathrm{d} V_{h_{K}}(x) \\
\leq & \varepsilon\left(\left\|u_{k}\right\|_{H_{1}^{2}}+\|u\|_{H_{1}^{2}}\right)\left\|u_{k}-u\right\|_{H_{1}^{2}} \\
& +m_{\varepsilon}\|\kappa\|_{L^{\infty}}\left(\left\|u_{k}\right\|_{L^{p}}^{p-1}+\|u\|_{L^{p}}^{p-1}\right)\left\|u_{n}-u\right\|_{L^{p}}
\end{aligned}
$$

Since $\varepsilon>0$ is arbitrary small and $u_{k} \rightarrow u$ strongly in $L^{p}\left(B^{n}(1)\right)$, the last expression tends to zero.

Moreover, relation (2.8) implies that

$$
\begin{aligned}
\mathcal{E}_{r}\left(u-u_{k}\right) & =\int_{B^{n}(1)} F_{a}^{* 2}\left(D u(x)-D u_{k}(x)\right) \mathrm{d} V_{F_{a}}(x) \\
& \leq\left(\frac{1+a}{1-a}\right)^{2} \int_{B^{n}(1)}\left(D u(x)-D u_{k}(x)\right)\left(\nabla_{F_{a}} u(x)-\nabla_{F_{a}} u_{k}(x)\right) \mathrm{d} V_{F_{a}}(x) .
\end{aligned}
$$

Therefore, $\mathcal{E}_{r}\left(u-u_{k}\right) \rightarrow 0$ as $k \rightarrow \infty$, which means in particular (see Remark 3.1) that $\left\{u_{k}\right\}$ converges strongly to $u$ in $H_{r}\left(B^{n}(1)\right)$.

STEP 5. (First solution) On account of the assumption made on $\kappa$ and (g2), there exists a truncation function $u_{0} \in H_{r}\left(B^{n}(1)\right) \backslash\{0\}$ such that $\mathcal{G}_{r}\left(u_{0}\right)>0$. Thus, we may define

$$
\tilde{\lambda}=\inf _{\substack{u \in H_{r}\left(B^{n}(1)\right) \\ \mathcal{G}_{r}(u)>0}} \frac{\mathcal{E}_{r}(u)}{2 \mathcal{G}_{r}(u)} .
$$

By (4.3), we clearly have that $0<\tilde{\lambda}<\infty$. If we fix $\lambda>\tilde{\lambda}$, there exists $\tilde{u}_{\lambda} \in H_{r}\left(B^{n}(1)\right)$ with $\mathcal{G}_{r}\left(\tilde{u}_{\lambda}\right)>0$ such that $\lambda>\frac{\mathcal{E}_{r}\left(\tilde{u}_{\lambda}\right)}{2 \mathcal{G}_{r}\left(\tilde{u}_{\lambda}\right)} \geq \tilde{\lambda}$. Thus,

$$
c_{\lambda}^{1}:=\inf _{H_{r}\left(B^{n}(1)\right)} \mathcal{R}_{\lambda} \leq \mathcal{R}_{\lambda}\left(\tilde{u}_{\lambda}\right)=\frac{1}{2} \mathcal{E}_{r}\left(\tilde{u}_{\lambda}\right)-\lambda \mathcal{G}_{r}\left(\tilde{u}_{\lambda}\right)<0
$$

Since $\mathcal{R}_{\lambda}$ is bounded from below and verifies the Palais-Smale condition, the number $c_{\lambda}^{1}$ is a critical value of $\mathcal{R}_{\lambda}$, i.e., there exists $u_{\lambda}^{1} \in H_{r}\left(B^{n}(1)\right)$ such that $\mathcal{R}_{\lambda}\left(u_{\lambda}^{1}\right)=c_{\lambda}^{1}<0$ and $\mathcal{R}_{\lambda}^{\prime}\left(u_{\lambda}^{1}\right)=0$. In particular, $u_{\lambda}^{1} \neq 0$.

SteP 6. (Second solution) Fix $\lambda>\tilde{\lambda}$. Applying (4.5) with the choice $\varepsilon:=$ $\frac{\left(1-a^{2}\right)^{\frac{n+1}{2}}}{2(\lambda+1)(1+a)^{2}}$, it follows that

$$
\begin{aligned}
\mathcal{R}_{\lambda}(u) & =\frac{1}{2} \mathcal{E}_{r}(u)-\lambda \mathcal{G}_{r}(u) \\
& \geq \frac{\left(1-a^{2}\right)^{\frac{n+1}{2}}}{4(1+a)^{2}}\|u\|_{K}^{2}-\lambda \frac{m_{\lambda}}{p}\|\kappa\|_{L^{\infty}} S_{p}^{p}\|u\|_{H_{1}^{2}}^{p}
\end{aligned}
$$


where $p \in\left(2,2^{*}\right)$ and $m_{\lambda}=m_{\varepsilon}>0$. Let

$$
\rho_{\lambda}=\min \left\{\left\|\tilde{u}_{\lambda}\right\|_{H_{1}^{2}},\left(\frac{\left(1-a^{2}\right)^{\frac{n+1}{2}}}{8 \lambda\|\kappa\|_{L^{\infty}} S_{p}^{p} m_{\lambda}(1+a)^{2}\left(1+\frac{4}{(n-1)^{2}}\right)}\right)^{\frac{1}{p-2}}\right\} .
$$

The latter estimate and Step 5 shows that

$$
\inf _{\|u\|_{H_{1}^{2}}=\rho_{\lambda}} \mathcal{R}_{\lambda}(u)=\eta_{\lambda}>0=\mathcal{R}_{\lambda}(0)>\mathcal{R}_{\lambda}\left(\tilde{u}_{\lambda}\right),
$$

i.e., the functional $\mathcal{R}_{\lambda}$ has the standard mountain pass geometry. According to Step 4, one may apply the mountain pass theorem, showing that there exists $u_{\lambda}^{2} \in H_{r}\left(B^{n}(1)\right)$ such that $\mathcal{R}_{\lambda}^{\prime}\left(u_{\lambda}^{2}\right)=0$ and $\mathcal{R}_{\lambda}\left(u_{\lambda}^{2}\right)=c_{\lambda}^{2}$, the number $c_{\lambda}^{2}$ being characterized by

$$
c_{\lambda}^{2}=\inf _{\gamma \in \Gamma} \max _{t \in[0,1]} \mathcal{R}_{\lambda}(\gamma(t))
$$

where

$$
\Gamma=\left\{\gamma \in C\left([0,1] ; H_{r}\left(B^{n}(1)\right)\right): \gamma(0)=0, \gamma(1)=\tilde{u}_{\lambda}\right\} .
$$

Since $c_{\lambda}^{2} \geq \inf _{\|u\|_{H_{1}^{2}}=\rho_{\lambda}} \mathcal{R}_{\lambda}(u)>0$, it is clear that $0 \neq u_{\lambda}^{2} \neq u_{\lambda}^{1}$. Since $g(s)=0$ for every $s \leq 0$, both solutions $u_{\lambda}^{1}$ and $u_{\lambda}^{2}$ are non-negative, following from (4.1). This concludes the proof.

Remark 4.1. (i) The case $a=1$ (Funk model) is not well understood, since the set $W_{0}^{1,2,1}\left(B^{n}(1)\right)$ is not a vector space over $\mathbb{R}$. However, we believe that variational problems can also be treated within this context by using elements from the theory of variational inequalities involving the indicator function associated with the closed convex cone $W_{0}^{1,2,1}\left(B^{n}(1)\right)$ in $L_{1}^{2}\left(B^{n}(1)\right)$, see [9, Section 2].

(ii) For simplicity of the presentation, we considered elliptic problems involving sublinear terms at infinity. The above variational arguments seem to work also for elliptic problems involving the Finsler-Laplace operator $\boldsymbol{\Delta}_{F_{a}}, a \in[0,1)$, and superlinear or oscillatory nonlinear terms, see e.g. Kristály [8].

Acknowledgment. A. Kristály is supported by a grant of the Romanian National Authority for Scientific Research, CNCS-UEFISCDI, "Symmetries in elliptic problems: Euclidean and non-Euclidean techniques", project no. PN-II-ID-PCE-2011-3-0241.

\section{REFERENCES}

[1] D. Bao, S. S. Chern, Z. Shen, Introduction to Riemann-Finsler Geometry, Graduate Texts in Mathematics, 200, Springer Verlag, 2000.

[2] M. Bhakta, K. Sandeep, Poincaré-Sobolev equations in the hyperbolic space. Calc. Var. Partial Differential Equations 44 (2012), no. 1-2, 247-269.

[3] X. Cheng, Z. Shen, Finsler geometry. An approach via Randers spaces. Science Press Beijing, Beijing; Springer, Heidelberg, 2012.

[4] S. S. Chern, Z. Shen, Riemann-Finsler geometry. Nankai Tracts in Mathematics, 6. World Scientific Publishing Co. Pte. Ltd., Hackensack, NJ, 2005.

[5] H. Federer, W. H. Fleming, Normal and integral currents. Ann. of Math. 72(1960), 458-520.

[6] Y. Ge, Z. Shen, Eigenvalues and eigenfunctions of metric measure manifolds. Proc. London Math. Soc. (3) 82 (2001), no. 3, 725-746. 
[7] E. Hebey, Nonlinear analysis on manifolds: Sobolev spaces and inequalities. Courant Lecture Notes in Mathematics, 5. New York University, Courant Institute of Mathematical Sciences, New York; American Mathematical Society, Providence, RI, 1999.

[8] A. Kristály, Detection of arbitrarily many solutions for perturbed elliptic problems involving oscillatory terms. J. Differential Equations 245 (2008), no. 12, 3849-3868.

[9] A. Kristály, V. Rădulescu, Cs. Varga, Variational Principles in Mathematical Physics, Geometry, and Economics, Cambridge University Press, Encyclopedia of Mathematics and its Applications, No. 136, 2010.

[10] H.-B. Rademacher, A sphere theorem for non-reversible Finsler metrics. Math. Ann. 328 (2004), no. $3,373-387$.

[11] G. Randers, On an asymmetrical metric in the fourspace of general relativity. Phys. Rev. (2) 59 (1941), 195-199.

[12] S. Ohta, K.-T. Sturm, Heat flow on Finsler manifolds. Comm. Pure Appl. Math. 62 (2009), no. 10, $1386-1433$.

[13] Z. Shen, Lectures on Finsler geometry. World Scientific Publishing Co., Singapore, 2001.

[14] Z. Shen, The non-linear Laplacian for Finsler manifolds. The theory of Finslerian Laplacians and applications, 187-198. Mathematics and Its Applications, 459. Kluwer, Dordrecht, The Netherlands, 1998. 